\section{O6 (continued)}

to participating in physical activity. In the African American community, beauty salons have historically been a trusted environment for candid talk about a variety of issues.

Description: Healthy Diva Beauty Salon Project recruited 109 African American woman from 15 salons in San Diego, California to participate in the "60-Day Healthy Diva Challenge." The intervention included the following components: Stylin' For Life magazine, Mirror Messages, two meet-up groups, text messaging program and a Healthy Diva Challenge blog. The evaluation included a pre-post survey analysis of participant's change, including a Personal Health Assessment, and specific feedback on the intervention.

Evaluation: Participating women responded positively to the "Healthy Diva" brand, were excited to join the challenge, enjoyed the program, and made positive changes in their diet and physical activity behaviors. Specifically, participants increased total fruit and vegetable consumption, decreased consumption of sugar sweetened beverages, increased availability of fruits and vegetables at home, and increased their exercise.

Conclusions and Implications: The Healthy Diva Beauty Salon Project was a successful pilot project for participating African American women. Expanding the social media presence, specifically a stronger presence on Facebook and additional support between meet-ups would be key components when scaling up this project.

Funding: California Department of Public Health.

\section{Blueprint for Creative/Innovative Success in Nutrition Education: What Works and Future Strategy of an Online Graduate Program}

Diane Dembicki,PhD,LMT, CYT, dembicki@adelphi.edu, Adelphi University, College of Nursing \& Public Health, 1 South Avenue, Garden City, NY 11530

Objective: The results of successful best practices and high impact teaching strategies of a creative and innovative master's program in nutrition education are shared. Development, implementation, and future plans are highlighted. This is for consideration of using a proven format-online-for student success as indicated by student engagement and satisfaction as well as professor evaluations of student learning.

Target Audience: The target audience includes current and future educators, program developers, and practitioners, as well as students of nutrition who want an alternative to on-the-ground classrooms. This in turn can help meet demand for capacity building in nutrition.

Theory, Prior Research, Rationale: These best practices-what works-are based in part from preliminary work by the Society for Nutrition Education and Behavior on nutrition education competencies to the Sloan Consortium on online education, from Kuh's high impact practices to a new strategy-gamification-by Sheldon and Kapp.

Description: A brief history is illustrated of the course development, successful online teaching techniques prac- ticed, and a cutting-edge high impact strategy recently developed to continue creativity and innovation.

Evaluation: The courses and teaching techniques were initially evaluated by internal University committees, an external reviewer, and a State Education Department. Once implemented, each course (8 so far) was evaluated by enrolled students $(n=51)$ through formative, summative, and post course surveys.

Conclusions and Implications: Results indicate high satisfaction and show that asynchronous collaborative learning in a high-tech format is preferred to other techniques and is effective in student success. This particular narrative can be a blueprint for current and potential programs to take nutrition education to the next level and into the future.

Funding: Adelphi University.

\section{Teaching Parents About Responsive Feeding Through a Vicarious Learning Video}

TraceyLedoux,PhD, RDN, TALedoux@uh.edu, University of Houston, 3855 Holman Street, Garrison Gym Room 104, Houston, TX 77204; J. Robinson, BS;

T. Baranowski, PhD, Baylor College of Medicine;

D. O'Connor, PhD, University of Houston

Objective: Responsive feeding practices (FPs) promote healthy weight and eating behaviors among preschoolers (2-5 years). This study aimed to test a vicarious learning video on parent responsive feeding knowledge and beliefs. Design, Setting and Participants: Participated via the web. Parents of preschoolers. The 20-minute experimental video featured children responding positively (e.g., eating vegetables) to responsive FPs and negatively (e.g., refusing food) to unresponsive FPs. The control video was about the education system.

Outcome Measures and Analyses: Knowledge and beliefs about unresponsive FPs (encouragement, control, pressure, weight-based and fat restriction, and instrumental and emotional feeding) and responsive FPs (covert control and monitoring) were measured one week before and immediately after intervention. A series of one-way ANOVAs were used to examine group differences on outcomes.

Results: Participants $(\mathrm{n}=50)$ were white $(74 \%)$, married (96\%), and $90 \%$ had at least a college degree, and $88 \%$ had income greater than $\$ 50 \mathrm{~K} / \mathrm{yr}$. Knowledge $(\mathrm{p}=.03)$ increased and beliefs about some unresponsive FP's [encouragement $(\mathrm{p}=.03)$, control $(\mathrm{p}=.02)$, pressure $(\mathrm{p}=.01)$, and weight-based restriction $(\mathrm{p}=.003)]$ decreased more among experimental than control parents. Beliefs about remaining FP's did not significantly change (p-values were greater than .05).

Conclusions and Implications: Responsive feeding knowledge and beliefs that were changed with the vicarious learning video were related to responsive FPs that were directly portrayed in the video. Vicarious learning may not be the optimal strategy for changing beliefs about subtle FPs (e.g., covert control) which are difficult to model. Vicarious learning videos may be an

Continued on page $S 4$ 